\title{
OPEN Defensive freezing and its relation to approach-avoidance decision-making under threat
}

\author{
Felix H. Klaassen ${ }^{1 凶}$, Leslie Held ${ }^{1,2}$, Bernd Figner ${ }^{1,2}$, Jill X. O’Reilly ${ }^{3}$, Floris Klumpers ${ }^{1,2}$, \\ Lycia D. de Voogd ${ }^{1}$ \& Karin Roelofs ${ }^{1,2}$
}

Successful responding to acutely threatening situations requires adequate approach-avoidance decisions. However, it is unclear how threat-induced states-like freezing-related bradycardia-impact the weighing of the potential outcomes of such value-based decisions. Insight into the underlying computations is essential, not only to improve our models of decision-making but also to improve interventions for maladaptive decisions, for instance in anxiety patients and first-responders who frequently have to make decisions under acute threat. Forty-two participants made passive and active approach-avoidance decisions under threat-of-shock when confronted with mixed outcome-prospects (i.e., varying money and shock amounts). Choice behavior was best predicted by a model including individual action-tendencies and bradycardia, beyond the subjective value of the outcome. Moreover, threat-related bradycardia (high-vs-low threat) interacted with subjective value, depending on the action-context (passive-vs-active). Specifically, in action-contexts incongruent with participants' intrinsic action-tendencies, stronger bradycardia related to diminished effects of subjective value on choice across participants. These findings illustrate the relevance of testing approach-avoidance decisions in relatively ecologically valid conditions of acute and primarily reinforced threat. These mechanistic insights into approach-avoidance conflict-resolution may inspire biofeedback-related techniques to optimize decision-making under threat. Critically, the findings demonstrate the relevance of incorporating internal psychophysiological states and external action-contexts into models of approach-avoidance decision-making.

In the face of threat, it is extra important to weigh the value of potential outcomes of our decisions to optimally deal with the potentially harming situation. Imagine just overcoming the fear to ask for a salary raise but finding your superior in a bad mood. How do we weigh a possible detrimental outcome of a scolding against a beneficial outcome of a better income? And how do we override the initial tendency to actively back-off or passively avoid the situation? While the anticipated outcomes of the decision play a decisive role in resolving such approach-avoidance conflicts, the presence of threat triggers a psychophysiological defensive state that may inform this decision ${ }^{1,2}$. Understanding how transient psychophysiological states affect our behavior is essential to advance models of human decision-making as well as to improve interventions for anxiety patients or first responders, who are constantly forced to make decisions under threat ${ }^{3-8}$. Yet, existing models of decision-making do not account for defensive threat states. Here we aim to bridge this gap between affective and decision sciences. Using a novel approach-avoidance conflict task, we test the link between threat-induced psychophysiological states and value-based approach-avoidance conflict decisions and their action-implementation.

Under acute threat, activation of the autonomic nervous system triggers a defense cascade ${ }^{2,9}$. Whereas noradrenergically-driven sympathetic upregulation is associated with increased heart rate and muscular tone enabling active fight-or-flight responses, concurrent cholinergically-driven parasympathetic dominance under proximal threat results in a net heart rate deceleration (i.e., bradycardia) and motor inhibition characteristic of a freezing response $^{10-13}$. Freezing is a universal defensive response to upcoming threat observed across many species ${ }^{14-16}$. In anticipation of threat, it serves as a temporary break on the cardiac and motor system, whereby its attention and perception enhancing properties are thought to affect subsequent fight-or-flight decisions through enhanced risk assessment ${ }^{1,17-20}$. In rodents and other animals, threat-anticipatory freezing is generated by projections from the

${ }^{1}$ Radboud University, Donders Institute for Brain, Cognition and Behaviour, Nijmegen, The Netherlands. ${ }^{2}$ Radboud University, Behavioural Science Institute, Nijmegen, The Netherlands. ${ }^{3}$ Wellcome Centre for Integrative Neuroimaging, Department of Experimental Psychology, University of Oxford, Oxford, UK. অemail: f.klaassen@ donders.ru.nl 
central nucleus of the amygdala to the midbrain periaqueductal gray (PAG), which in turn effectuates immobility via medullar projections to spinal cord motor neurons and bradycardia via the vagus nerve $\mathrm{e}^{10,21-23}$. Recent work in humans confirmed similar neural mechanisms underlying the human freezing response ${ }^{6,24}$. Importantly, in humans, this state of immobility and bradycardia has been associated with preferred visual perception of low spatial frequency features ${ }^{25}$, which is important for fast threat detection and facilitated by direct amygdala-visual cortex projections during freezing ${ }^{26}$. Together with the fact that freezing is stronger when active responding is possible $^{27}$, and that stronger freezing is associated with faster subsequent responding ${ }^{6}$, this shows that freezing may play an important role in decision-making under threat. It is however unclear how freezing affects the decision-making process.

One possibility is that freezing affects approach-avoidance conflict decisions by acting on the subjective value computations underlying the approach-avoidance conflict. So far, however, no studies have incorporated freezing (or the associated bradycardia) in value-based models of approach-avoidance conflict decisions. Previous work using gamble-based and foraging-like paradigms has resulted in valuable formal models of how individuals resolve choice conflict under outcome uncertainty by typically contrasting monetary gains versus losses ${ }^{28-31}$. Given the absence of formal threat induction and concurrent psychophysiological states, such paradigms may however not generalize to real-life conflict situations that involve anxious emotional states ${ }^{32}$. Only few studies have incorporated threat into approach-avoidance conflicts by contrasting (monetary) rewards with receiving electric shocks $s^{33-35}$ or by contrasting threat of shock with total safety ${ }^{36}$. Because these studies did not take into account the psychophysiological state of the decision-maker in their value-based decision models, it remains unclear whether such states are incorporated into the computation of the subjective value of the potential outcomes that underlies the decision. If freezing feeds into such outcome value computations, this could affect the decision by biasing the conflict towards increased avoidance ${ }^{37}$.

A second option is that the impact of freezing on the subjective value of approach-avoidance conflict decisions depends on whether an action is required. Indeed, previous studies ${ }^{27,38}$ found that freezing was reduced in situations where no action could be taken. However, because passive paradigms typically do not involve decision-making, the role of freezing in situations that offer the possibility for passive approach-avoidance remains unclear. Making a distinction between passive and active approach-avoidance decisions is especially relevant in light of profound individual differences in passive versus active avoidance strategies across different types of anxiety disorders ${ }^{39}$, for which an explanatory human model is largely lacking. Animal models have shown that freezing-induced action inhibition can hamper the opportunity for active avoidance ${ }^{15,40-42}$. However, more recent work in humans ${ }^{18,27,43}$ has shown that freezing is associated with action preparation. Therefore, how freezing affects approach-avoidance conflict decisions under threat may depend on the action context.

To test these hypotheses, we developed the passive-active approach-avoidance Task (PAT, Fig. 1), in which participants are required to integrate varying monetary and shock amounts into an approach-avoidance decision in both passive and active action contexts. Action contexts were created by manipulating the movement direction of the to be approached/avoided target (i.e., either away from or towards the participant icon). We operationalized freezing in the anticipation of the decision by means of heart rate deceleration (i.e., bradycardia). In addition, we assessed body sway (i.e., postural freezing) to confirm bradycardia as part of the threat-anticipatory freezing response in our novel task using a stabilometric force platform ${ }^{6}$. First, we assessed the ability of the task to induce approach-avoidance conflict by testing effects of reward and punishment on passive and active approach-avoidance conflict decisions using mixed-effects model analyses for choice behavior and response time. Then, we applied computational modeling to investigate whether bradycardia interacts with the subjective value of avoid versus approach choices, and/or with individuals' context-dependent passive versus active action tendencies.

\section{Results}

Task effects on passive and active approach-avoidance decision-making. Overall, the PAT showed the expected effects of potential money and shock amounts on choice behavior. A Bayesian generalized mixed-effects model showed that participants approached the targets significantly more as the amount of money that could be earned increased, and avoided significantly more as the number of shocks that could be received increased $\left(\mathrm{B}_{\text {money }}=1.41,95 \% \mathrm{CI}[1.17,1.70], p p_{<0}<0.001 ; \mathrm{B}_{\text {shocks }}=-1.08,95 \%\right.$ CI $[-1.38,-0.80], p p_{>0}<0.001$; note that effects were considered significant if the $95 \%$ CI did not include 0 , which corresponds to a posterior probability $(p p)$ value $<0.025$, see "Methods" section). Moreover, the effects of the amount of money and shocks on choice behavior interacted significantly $\left(B_{\text {money:shocks }}=0.33,95 \% \mathrm{CI}[0.15,0.51], p p_{<0}<0.001\right)$. Thus, with increasing amounts of potential money the effect of the shocks on avoidance behavior reduced, and with lower amounts of money the avoidance-inducing effects of high threat increased. Together, these results show that our new task is successful in evoking trade-offs between varying amounts of money and shocks (Fig. 2a).

Importantly, while there were no main effects of action context on approach-avoidance choices (Fig. 2b; $\left.\mathrm{B}_{\text {actioncontext }}=-0.11,95 \% \mathrm{CI}[-0.33,0.10]\right)$, an interaction effect with the potential money and shock amounts showed that the effect of the amount of money and shocks on choice behavior was smaller in the passive action context compared to the active action context $\left(B_{\text {actioncontext:money }}=-0.33,95 \% \mathrm{CI}[-0.47,-0.20]\right.$; $\left.\mathrm{B}_{\text {actioncontext:shocks }}=0.26,95 \% \mathrm{CI}[0.15,0.38]\right)$. More specifically, higher money amounts increased approach choices more strongly in active approach trials than passive approach trials, while higher shock amounts increased avoidance choices more strongly in active compared to passive trials (Fig. 2b). This appeared to be driven by a significant invigoration of active responses by increased money amounts, and by an inhibition of action by higher shock amounts (see Supplementary Results for details). A similar pattern of action invigoration and inhibition by money and shock, respectively, was observed in the response times (see Supplementary Fig. S1). Overall, while we had no explicit expectations on the effect of reward and punishment on action, these findings suggest 


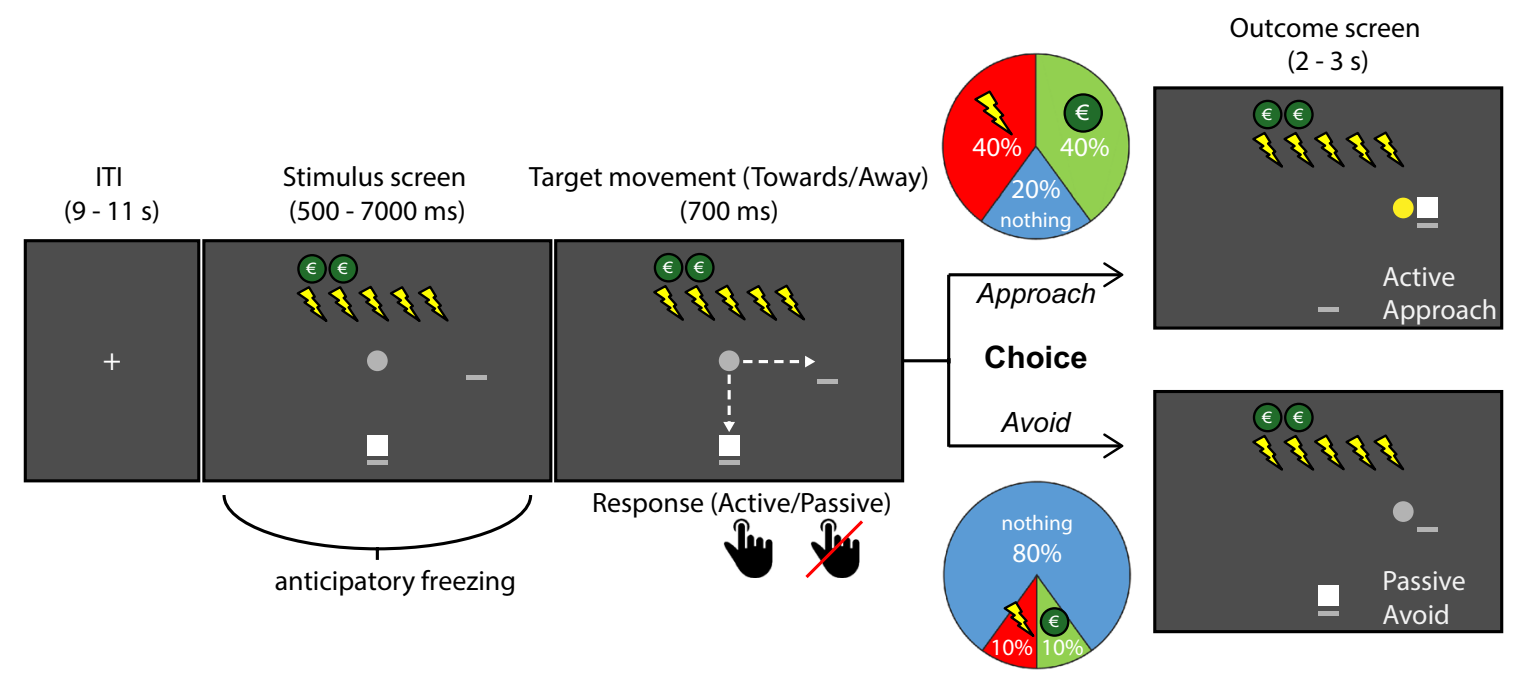

Figure 1. Timeline of an example trial in the Passive-active Approach-avoidance Task (PAT). After presentation of the trial-specific potential money and shock amounts (in this example 5 shocks and 2 Euro), the participant (white square) is asked to approach or avoid the target (gray circle) as it gradually moves either toward or away from the player (respectively passive vs. active action context, shown here=active). Depending on the action context, participants could give both passive and active approach-avoidance responses by either pressing a button (active) or not pressing the button (passive) during the target movement window. At the end of the trial the participant probabilistically received either shocks, money, or no outcome. Approach was associated with a high chance of receiving the shocks (40\%) or the money (40\%), and small chance of receiving nothing (20\%), while avoid responses led to a high chance of receiving nothing $(80 \%)$ and a low chance of receiving either the money (10\%) or shocks (10\%; see colored pie charts). After the target movement, the outcome of the trial was also indicated by a change of the target color into either green (money) or yellow (shocks), or no change (no outcome), and shocks were paid out immediately during that color change. Note: The dashed arrow lines were not present in the actual experiment.

a

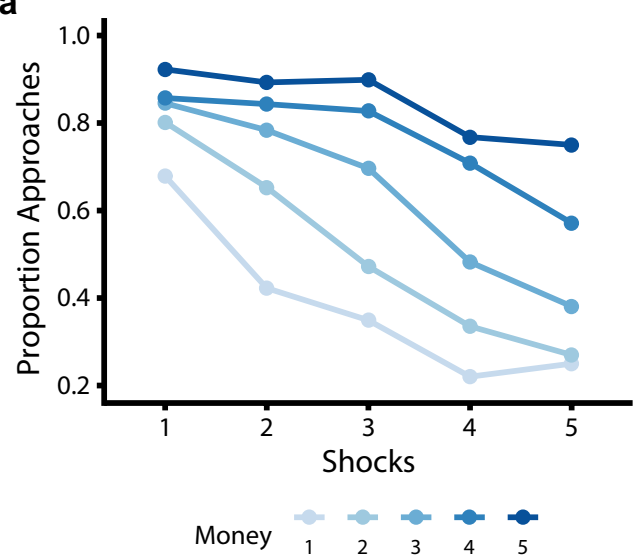

b

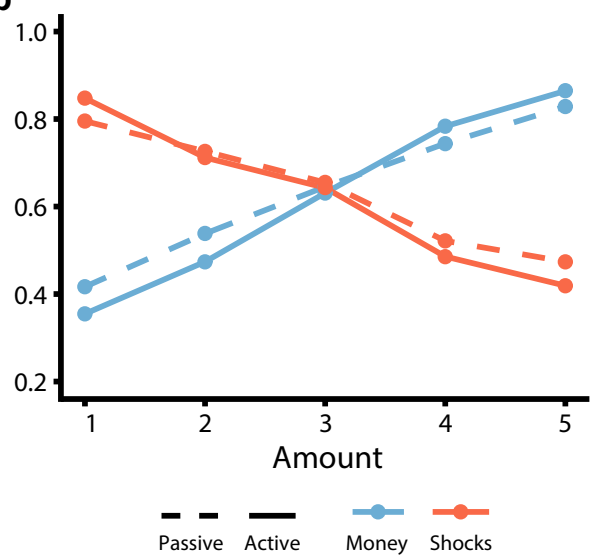

Figure 2. Effects of potential money and shocks and passive/active trial context on the proportion of approach choices. (a) Participants approach more with higher money amounts, and approach less with higher shock amounts. (b) Moreover, approach choices are more often active with increased money amounts and more often passive with higher shock amounts. Line types reflect active (solid) versus passive (dashed) approach conditions.

that increasing potential reward invigorates active compared to passive choices whereas increasing threat leads to more passive (compared to active) choices.

Psychophysiological dynamics of decision-anticipatory freezing. Next, we verified whether our threat manipulation (i.e., number of shocks) impacted bradycardia in the decision-anticipatory time window. Therefore, we ran a separate Bayesian generalized mixed-effects model with trial-by-trial bradycardia (HR) as dependent variable and continuous predictors money, shocks, their interaction, and body sway (BS). The latter measure was added to confirm the relationship between bradycardia with body sway as an index of freezing 
a

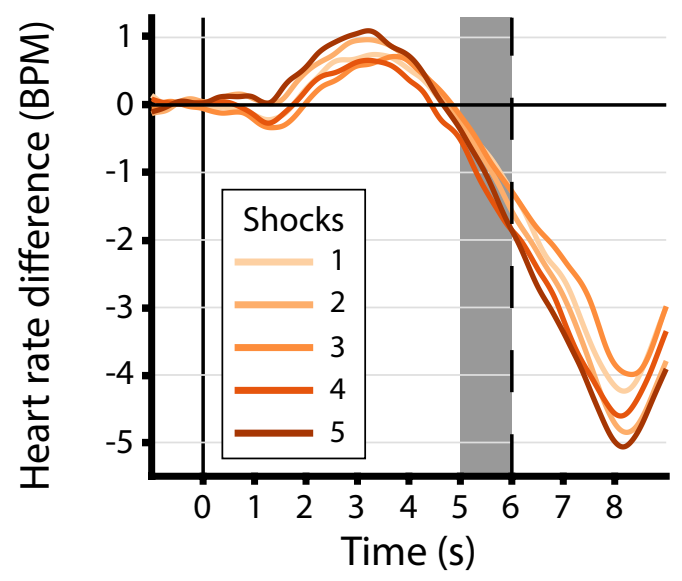

b

d

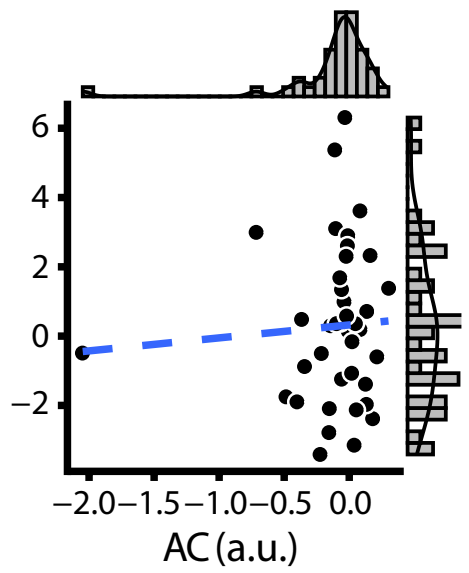

g

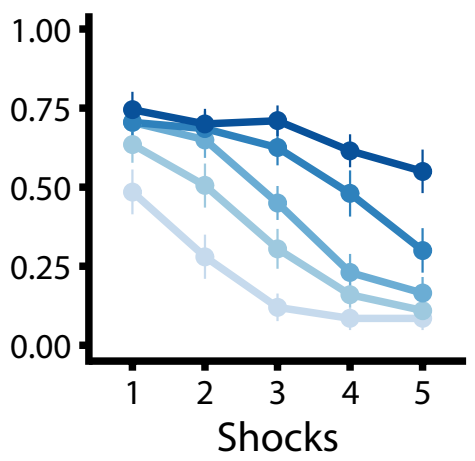

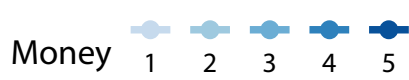

$\frac{n}{ \pm}$
$\frac{\pi}{0}$
$\frac{0}{0}$
$\frac{c}{0}$
$\frac{u}{0}$
$\frac{0}{2}$
Freeze $=$ Bradycardia

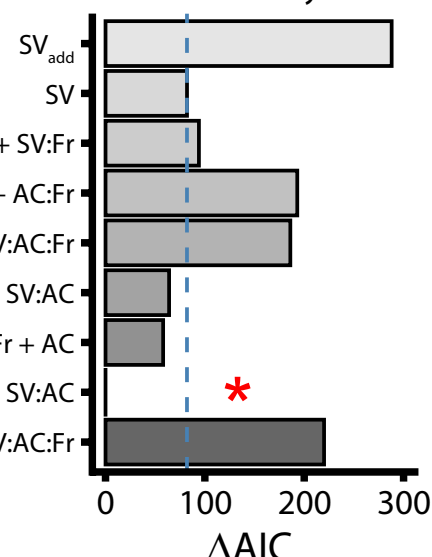

e

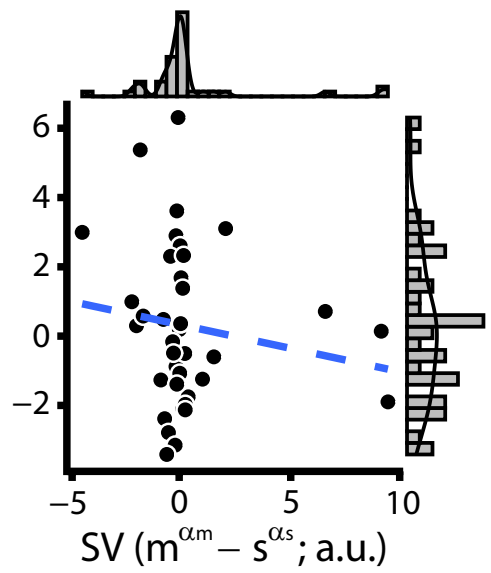

h

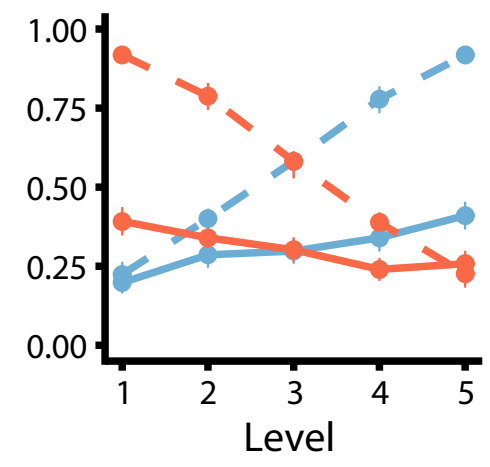

$-0-$
Passive Active Money Shocks 
4Figure 3. Heart rate and computational modeling results. Panel (a) depicts the average trial time course of baseline corrected heart rate (HR) as a function of the varying shock amounts, with the gray shaded area representing the time window of interest used in all analyses. The vertical dashed line represents the earliest possible target movement onset. Model comparison of various freeze models (b) showed that the best fitting model included the subjective value (i.e., potential money and shock amounts), bradycardia, the action context, and the interaction between the subjective value and action context parameters (indicated with a red asterisk). Additionally, on the subject level, stronger threat-related bradycardia (i.e., high vs. low threat) was related to the integration of action context (AC) and subjective value (SV) for approach-avoidance choices (c), but not with $\mathrm{AC}$ or SV alone (d,e). Simulations show that higher values of this interaction parameter led to a diminished effect of subjective value on choice in situations incongruent with passive/active response tendencies $(\mathbf{f}-\mathbf{h}$; for brevity, only simulations of passive 'participants' with a positive SV:AC interaction are shown here, see Supplementary Information for the full results). Model AIC scores are plotted as the difference from the best fitting model $(\triangle \mathrm{AIC})$; lower AIC scores indicate better fit. The blue dashed line $(\mathbf{b})$ represents the reference model's fit and the red asterisk indicates the overall winning model. Solid vs. dashed regression lines in (c-e) reflect significant versus non-significant relationships. Error bars in $(\mathbf{f}-\mathbf{h})$ represent one standard error of the mean $(\mathrm{SEM}) . \mathrm{SV}=$ subjective value, $\mathrm{AC}=$ action context, $\mathrm{Fr}=$ umbrella term for freeze indices: All models were fitted with either bradycardia or body sway as freeze index, see Supplementary Information; colons (:) denote interactions.

in the context of our novel value-based decision task. Here, we indeed observed a significant general deceleration of the average heart rate signal during the anticipation phase relative to the $1 \mathrm{~s}$ pre-trial baseline (Fig. 3a, $\left.\mathrm{B}_{\text {Intercept }}=-1.01,95 \% \mathrm{CI}[-1.73,-0.31], p p_{>0}<0.003\right)$. This deceleration was not further moderated by money $\left(95 \%\right.$ CI $\left.[-0.09,0.43], p p_{<0}=0.1\right)$ or shock amounts $\left(95 \%\right.$ CI $\left.[-0.38,0.19], p p_{>0}=0.25\right)$. Importantly, bradycardia was positively related to body sway reductions, confirming a trial-by-trial correlation between anticipatory bradycardia and movement cessation also found in previous freezing literature ${ }^{8,43-45}\left(\mathrm{~B}_{\mathrm{BS}}=0.61,95 \% \mathrm{CI}[0.81\right.$, $\left.1.08], p p_{<0}<0.003\right)$. To further explore the effect of the varying shock amounts on heart rate signal dynamics, in a data-driven approach we tested whether the steepness of the slope of the heart rate between the initial peak $( \pm 3 \mathrm{~s})$ and subsequent trough $( \pm 8 \mathrm{~s})$ (see Fig. 2a) was affected by the number of shocks. Indeed, a mixed model on the slope revealed that higher potential shock amounts led to steeper negative slopes in the heart rate signal (i.e., stronger deceleration; $\mathrm{B}_{\mathrm{HRslope}}=-0.37,95 \% \mathrm{CI}[-0.53,-0.02], p p_{>0}=0.018$ ). This, together with increased skin conductance responses as a function of increased shock amounts (see Supplementary Fig. S6), indicated that participants were psychophysiologically affected by our threat manipulation.

Interestingly, and in line with previous studies showing action preparation during freezing ${ }^{6}$, trial-by-trial analyses indicated that stronger bradycardia was associated with faster responding regardless of choice (Supplementary Fig. S2; $\left.B_{\mathrm{HR}}=0.03,95 \% \mathrm{CI}[0.01,0.06], p p_{<0}<0.006\right)$. In addition, we found no relationship of bradycardia with approach-avoidance choices or passive vs. active responding, suggesting that-contrary to our hypotheses-trial-by-trial bradycardia is not directly related to increased avoidance or passive versus active responding.

Computational modeling of freezing in approach-avoidance decision-making. Next, we used computational modeling to investigate whether bradycardia interacts with the subjective value of the potential outcome of approach-avoidance choices (SV) or with the action context (AC). In our models, the subjective value term reflects the subjective influence of potential money and shock amounts on approach-avoidance choices, whereas the action context parameter AC makes use of the action contexts to capture individuals' action tendencies [i.e., their general tendency to respond passively $(\mathrm{AC}>0)$ or actively $(\mathrm{AC}<0)$ ]. We performed model comparison on various model candidates (Table 1) to investigate how bradycardia is most likely to interact with approach-avoidance choices.

This revealed that the model including the subjective value of the potential outcomes, bradycardia (as a main effect), the action context parameter $\mathrm{AC}$, and the interaction between the subjective value and the action context parameter (SV:AC) yielded the best fitting model (i.e., lowest AIC; red asterisk in Fig. 3b). Indeed, although we found no average main effect of passive-active trial contexts on choice behavior, accounting for individual differences in passive/active action tendencies and their interaction with the subjective value of money and shock amounts allows us to better explain approach-avoidance choices. As a check we performed the same model comparison with body sway as alternative freezing index which yielded the similar results and conclusions (see Supplementary Fig. S3).

While these model comparison findings provide some insight into how general heart rate indices of freezing are most likely to play a role in the decision-making process on the trial-by-trial level, the question remained whether threat-related freezing on the subject level would interact with the subjective value of the choice or the action context. Is the extent to which a person freezes in response to threat related to how they resolve approach-avoidance conflicts? According to our hypotheses, freezing could interact with subjective value $\left(m^{\alpha_{m}}-s^{\alpha_{s}}\right)$ or action implementation alone (AC parameter), but we also considered the possibility that it relates to how the subjective value interacts with passive versus active responses $\left(\beta_{\mathrm{SV}: \mathrm{AC}}\right)$. To test this, we performed exploratory correlations on the threat-induced individual differences in bradycardia (high vs. low threat, see Supplementary Methods) and the parameter estimates of the winning computational model (Fig. 3b). This threat-related bradycardia did not correlate to the action parameter AC or subjective value alone (both $p>0.1$ ), but did correlate with their interaction ( $\beta_{\mathrm{SV}: \mathrm{AC}}, \mathrm{Rs}=0.36, p=0.022$, uncorrected for 3 correlations; Fig. $\left.3 \mathrm{c}-\mathrm{e}\right)$.

To gain further understanding of the positive relationship between threat-related bradycardia and the $\beta_{\mathrm{SV}: \mathrm{AC}}$ parameter, we performed simulations of approach-avoidance choices for positive vs. negative values of the $\beta_{\mathrm{SV}: \mathrm{AC}}$ 


\begin{tabular}{|l|l|l|l|}
\hline & Model name & Extra parameters relative to the reference model & Number of free parameters \\
\hline 1 & $\mathrm{SV}_{\text {add }}$ & $\left(\right.$ no $\left.\beta_{\mathrm{m}: \mathrm{s}}\right)$ & 2 \\
\hline 2 & $\mathrm{SV} \dagger$ & - & 3 \\
\hline 3 & $\mathrm{SV}+\mathrm{SV}: \mathrm{Fr}$ & $\beta_{\mathrm{SV}: \mathrm{Fr}}$ & 4 \\
\hline 4 & SV $+\mathrm{AC}: \mathrm{Fr}$ & $\beta_{\mathrm{AC}: \mathrm{Fr}}, \mathrm{AC}$ & 5 \\
\hline 5 & $\mathrm{SV}+\mathrm{SV}: \mathrm{AC}: \mathrm{Fr}$ & $\beta_{\mathrm{SV}: \mathrm{AC}: \mathrm{Fr}} \mathrm{AC}$ & 5 \\
\hline 6 & $\mathrm{SV}+\mathrm{Fr}+\mathrm{SV}: \mathrm{AC}$ & $\beta_{\mathrm{Fr}}, \beta_{\mathrm{SV}: \mathrm{AC}}, \mathrm{AC}$ & 6 \\
\hline 7 & $\mathrm{SV}+\mathrm{Fr}+\mathrm{AC}$ & $\beta_{\mathrm{Fr}}, \mathrm{AC}$ & 5 \\
\hline 8 & $\mathrm{SV}+\mathrm{Fr}+\mathrm{AC}+\mathrm{SV}: \mathrm{AC}$ & $\beta_{\mathrm{Fr}}, \mathrm{AC}, \beta_{\mathrm{SV}: \mathrm{AC}}$ & 6 \\
\hline 9 & $\mathrm{SV}+\mathrm{SV}: \mathrm{Fr}+\mathrm{AC}: \mathrm{Fr}+\mathrm{SV}: \mathrm{AC}: \mathrm{Fr}$ & $\beta_{\mathrm{SV}: \mathrm{Fr}}, \beta_{\mathrm{AC}: \mathrm{Fr},} \beta_{\mathrm{SV}: \mathrm{AC}: \mathrm{Fr}}, \mathrm{AC}$ & 7 \\
\hline
\end{tabular}

Table 1. Name and parametrization of all candidate models. $\mathrm{m}=$ money; $\mathrm{s}=$ shocks; $\mathrm{AC}=$ action context; $\mathrm{SV}=$ subjective value, $\mathrm{Fr}=$ umbrella term for freeze indices: each model was fitted once with bradycardia and once with body sway (reported in supplements); $\mathrm{SV}_{\mathrm{add}}=$ additive $\mathrm{SV}$ model; $\uparrow$ reference model; colons (:) denote interactions.

parameter, both for 'participants' with passive and active response tendencies. These revealed that more positive $\beta_{\mathrm{SV}: A C}$ parameter values result in a diminished effect of potential money and shock amounts on choice behavior for active approach trials in passive participants $(\mathrm{AC}>0)$ and for passive approach trials in active participants $(\mathrm{AC}<0)$, and vice versa for negative $\beta_{\mathrm{SV}: \mathrm{AC}}$ values (Fig. $3 \mathrm{f}-\mathrm{h}$ and Supplementary Fig. S4). More generally, these simulations indicate that individuals with stronger threat-related bradycardia may differentially weigh the influence of potential reward and threat outcomes depending on the action context. When the action context does not match the internal action tendency, stronger threat-related bradycardia is associated with a diminished effect of subjective value on choice, whereas in congruent action contexts the effect of subjective value on choice remains relatively unaffected.

\section{Discussion}

This study aimed to bridge the fields of affective and decision sciences by systematically manipulating psychophysiological threat-states and action contexts during approach-avoidance conflict resolution. Three main findings contribute novel insights into decision-making under acute threat. First, at the behavioral level, participants were well able to trade-off money versus shocks and whereas high threat was associated with more passive responding, high reward induced more active responding. Interestingly, this motivation-related invigoration of action was also observed in the response times. Second, freezing-related bradycardia was related to faster responding but there was no direct relationship with choice or action. Third, approach-avoidance decisions were best predicted by a model that included not only the subjective value of the potential outcomes, but also incorporated bradycardia and the action context. Moreover, at the subject level, threat-related bradycardia (high vs. low threat) was positively correlated to the interaction between the action (passive/active) parameter and the subjective value. Specifically, stronger bradycardia was associated with an increasingly diminished effect of outcome subjective value on choice when the action context did not match individuals' passive/active action tendencies, signifying a potential relationship between freezing and the way decisions are made on the subject level. Together, these findings show that integration of psychophysiological states as well as action context into our decision models yields new mechanistic insight into how approach-avoidance conflict decisions are made under threat. This has implications for behavior in real-life threatening situations, such as for first-responders, anxiety patients, and anyone facing conflict-decisions in daily life (see Supplementary Information for a realworld example), suggesting that it may be beneficial to learn to control freezing-related states (e.g., using biofeedback ${ }^{46,47}$ or non-invasive brain stimulation techniques ${ }^{48}$ ) to help optimally weighing values in the decision. Given this relevance of approach-avoidance conflict decision-making for real-life threatening situations, these findings call for models of value-based decision-making that better integrate internal (e.g., value, psychophysiology, and action tendencies) and external (e.g., action context) components of the decision.

Anticipation of decisions was associated with freezing-like reactions, both in terms of heart rate deceleration (bradycardia) and body sway reduction. Following previous work ${ }^{8,43-45}$ and relevant for subsequent neuroimaging studies, we validated the heart rate measure showing a correlation to a concurrent bodily freezing measure (see Supplementary Fig. S3). Bradycardia did not interact with approach-avoidance conflict decisions directly, but seemed to rather act on the implementation of the required action as well as its interaction with subjective value.

Regarding the relation between freezing and action implementation, bradycardia was associated with significantly faster response times for subsequent action, supporting a role of freezing in action preparation ${ }^{11}$. Although few previous animal studies suggested freezing to be part of a general response inhibition ${ }^{15,40,41}$, our results are more in line with recent human literature consistently showing that across parameters (i.e., heart rate, body sway, and neural (midbrain PAG) activity), increased freezing magnitude is associated with faster subsequent action execution, without going at the expense of an increase in error rates ${ }^{6,27}$. This discrepancy is likely related to the fact that freezing in the animal literature is typically defined as the reduction or absence of movement ${ }^{40}$. The lack of bradycardia indices makes it harder to distinguish it from other passive defensive states: such as orienting and tonic and collapsed immobility ${ }^{2}$. Recent animal work that considered heart rate indeed found that bradycardia was related to immobility in anticipation of threat (to a conditioned cue) and not after (unconditioned) threat 
exposure $^{24}$. Also, in the animal literature freezing is often assessed in terms of response duration rather than magnitude of state-changes, confounding the relation with subsequent action. Future research using more precise translation between animal and human paradigms has the promise to assess similarities and discrepancies in the function of freezing across species ${ }^{49}$.

Importantly, individual approach-avoidance choices were best predicted by a model including not only the effects of the subjective value of the potential outcomes, but also bradycardia and the action context (active-vspassive). This is in line with the notion that freezing plays a role in decision-making under threat ${ }^{1,11,37}$, though on the trial level this does not seem to take place through interaction with outcome value computations or action tendencies. Interestingly, on the subject level, the relation between threat-related bradycardia and subjective value depended on the congruency between the passive-active trial context and the participant's passive-active response tendency. Specifically, stronger threat-related bradycardia was associated with a diminished effect of subjective value on choice in situations that are incongruent with the individual's passive or active response tendency. This finding may be interpreted in line with previous notions that people with stronger threat-induced freezing show general upregulation of sensory system ${ }^{26}$ and better risk assessment ${ }^{1}$. Such upregulation may be hampered when counterintuitive actions have to be prepared. Speculatively, contexts that do not match some internal action tendency may put increased demand on cognitive control processes involved in allocation of resources needed to weigh decision-relevant information ${ }^{50-52}$. This context-dependency of the effect of threatrelated bradycardia on behavior also suggests that cognitive effects of freezing cannot be explained by a general increase in attention, which is in line with previous findings showing specific effects of freezing on features of visual perception (e.g., improved low spatial frequency visual perception at the expense of high spatial frequency ${ }^{25}$ ) as well as instrumental approach-avoidance actions (e.g., slowing of approach but not avoidance choices $^{37}$ ).

The notion that individual bias in passive versus active responding matters in the relation between freezing and subjective value may explain that the results from our mixed-effect models showed no average trial-by-trial relation between bradycardia and approach-avoidance choices. Likewise, Ly and colleagues ${ }^{37}$ found a relation between freezing and subsequent approach-avoidance decisions between-subjects but not within. Together, the between-subject nature of our freezing and action context findings may also imply that our freezing effects may partly reflect a trait-like effect, an interpretation that is further supported by genetic components previously identified for individual differences in freezing-tendencies ${ }^{24,53}$.

The relevance of considering active versus passive decisions during approach-avoidance conflict was not only supported by its interaction with bradycardia. Our computational modeling approach showed that the freeze model that accounted for individuals' passive-active response tendencies as well as its interaction with the subjective value of the outcomes, explained choice behavior significantly better than a standard subjective value model. Finally, the relevance of taking action context into account was supported in our task-validation mixed effects models approach, where it helped to reveal a clear pattern of reward-induced action invigoration: higher money amounts led to more active responses, whereas more potential shocks led to more passive responses. These findings fall in line with many studies in the go/no-go literature that demonstrate action invigoration and inhibition in the face of reward vs. threat ${ }^{54}$. Mimicking these choice effects, we additionally observed faster response times for approach choices for increased money amounts, and faster responses for avoid choices for increased shock amounts, suggesting motivation-related invigoration of action. Together, these finding show that it is relevant to incorporate psychophysiological states and action context into value-based models of approach-avoidance conflict decisions.

Some limitations should be considered when interpreting these findings. First, although freezing temporally preceded the choice behavior in our paradigm, we cannot make causal inferences on the role of freezing in decision-making. Such causality may be achieved in future studies, for example by stimulating the vagal nerve or by stimulating brain regions that are thought to be causally involved in the regulation of freezing-related bradycardia, such as deep brain stimulation of the $\mathrm{PAG}^{10,55}$. Second, although this is the first study testing approach-avoidance conflict resolution under relatively ecologically valid conditions of acute and primarily reinforced threat (threat-states being validated in all three psychophysiological measures), follow-up studies using ambulatory monitoring of psychophysiological states should test whether findings generalize to real-life decisions. Third, because the interpretations of the correlation between threat-related bradycardia and model parameters are based on an exploratory analysis, they should be regarded as hypothesis-generating rather than hypothesis-testing. Still, while provisional, these findings provide deeper insight into the relationship between freezing and decision-making which could be meaningfully interpreted using model simulations.

Based on our findings, we make some recommendations for future studies investigating the relationship between defensive (freezing) responses and decision-making under threat. First, it is important to measure approach-avoidance behavior under acute and primary threat (e.g., threat of shock), since that triggers the psychophysiological state also present in real-life anxiety-inducing conflict situations. Second, future studies should not only investigate how potential choice outcomes determine behavior, but also how this varies as a function of the psychophysiological state of the decision-maker and the context in which the decision takes place. Specifically, we hope that paradigms such as the one presented in this study will be able to measure and predict differences in passive and active avoidance tendencies observed in clinical populations ${ }^{39}$. Finally, investigations into the neural underpinnings of defensive freezing and its relation to decision-making under threat may point to which avenues of intervention are suitable and promising to counter maladaptive decision-making.

In conclusion, we present a novel paradigm in which we could capture how threat-related psychophysiological states such as freezing-related bradycardia are associated with passive and active approach-avoidance conflict resolution. Our results support the relevance of considering individuals' tendency to respond passively versus actively and the role of such action-related parameters in explaining choice behavior besides the subjective value associated with approach and avoidance. Second, although bradycardia may not be related to changes in approach-avoidance conflict decisions by directly affecting the subjective value of the potential outcomes, 
it interacts with how approach-avoidance conflict decisions are made on the individual level, coinciding with a diminished effect of the subjective value of the choice outcomes in situations that are incongruent with the dominant passive or active response tendency. Critically, our findings illustrate that it is important to incorporate concurrent external action contexts as well as internal psychophysiological states of the decider in models of approach-avoidance conflicts.

\section{Methods}

This study was preregistered on the Open Science Framework before data collection (https://osf.io/68e75/). All research activities were carried out in accordance with the Declaration of Helsinki and approved by the local ethics committee (Ethical Reviewing Board CMO/METC [Institutional Research Review Board] Arnhem-Nijmegen, CMO 2014/288) and conducted according to these guidelines and regulations (i.e., medical/scientific research).

Participants. A total of 42 participants (aged between 18 and 35 [ $\mathrm{M} \pm \mathrm{SD}=23.5 \pm 3.63]$; 24 females) were included in the study. An additional three participants were excluded due to technical issues $(n=2)$ and dizziness $(n=1)$. For the computational modeling analyses only, two (out of 42 ) participants were excluded due to a lack of variability in the choice data, meaning these analyses involved 40 participants (aged between 18 and $35[\mathrm{M} \pm \mathrm{SD}=23.28 \pm 3.46] ; 24$ females). Sample size was determined using a simulation-based power analysis ${ }^{56}$ (pilot sample of $n=5$ ) which revealed a sample size of 40 participants was sufficient to detect a small (i.e., odds ratio of $1.5^{57}$ ) bradycardia by shock interaction effect on choice with at least $80 \%$ power. Inclusion criteria were age (younger than 18 or older than 35), Dutch or English speaking, and right-handedness; exclusion criteria were self-reported current pregnancy, current or lifetime history of psychiatric, neurological, or cardiovascular disorder, endocrine illness, claustrophobia, plaster allergy, and self-reported high or low blood pressure. All participants gave written informed consent before participation, and were paid for participation (€16) plus bonus money contingent on their task choices (see below).

Experimental procedure. Participants came to the lab once for a $90 \mathrm{~min}$. assessment involving the Passive-active Approach-avoidance Task (PAT). Procedures started with performing a standardized 5-step shock work-up to calibrate the intensity of the electric shock which remained the same for the rest of the task (see "Peripheral stimulation and measurements" section).

Next, the participant stepped on the stabilometric force platform, read through the onscreen instructions, and performed practice trials. After the experimenter verified that instructions were understood, participants started the PAT paradigm which consisted of 150 trials divided up in 10 blocks ( \pm 5 min duration). Participants were encouraged to take breaks by stepping off the platform and sit down in between blocks to minimize the chance of exhaustion and potential dizziness due to prolonged standing still.

Afterwards, participants received one more electric shock and were asked to rate the intensity. Then, participants filled in a questionnaire in which they subjectively rated the attractiveness of all possible moneyshock-probability combinations (see Supplementary Fig. S5 for results). Finally, we administered the State Trait Anxiety Questionnaire $\left(\mathrm{STAI}^{58} ; M \pm S D=33.94 \pm 8.84\right)$ and the Beck Depression Inventory II (BDI-II ${ }^{59}$; $M \pm S D=6.38 \pm 4.92)$ to characterize the sample.

Experimental paradigm - passive-active approach-avoidance task (PAT). In the Passive-active Approach-avoidance Task (see Fig. 1), participants were instructed to make approach-avoidance decisions in response to a moving target that was associated with varying amounts of monetary rewards and shocks. On each trial, they either approached or avoided the target by positioning the player icon towards or away from the target, which probabilistically led to either money, electric shocks, or no outcome. Since extensive pilot work had indicated that in our task individuals were able to trade-off money (1-5 Euro) and shocks (1-5) (i.e., without inducing behavioral ceiling or floor effects, see Supplementary Fig. S7), we did not compute subject-specific money-to-shock indifference pairs; all participants received the same monetary offers. The monetary outcome of three randomly selected trials (max. €15) was paid out as a bonus fee. Finally, to be able to distinguish between active and passive approach-avoidance decisions, responses were given in two possible action context conditions (passive/active). This was determined by the movement direction of the target, which was either towards or away from the player icon. If the target moved away from the participant an approach decision involved an active response and avoid was passive. In case the target moved towards the participant, avoidance required an active response and approach was passive. Per trial, we recorded the participants' choice (approach/avoid), response type (passive/active), and response time. Psychophysiological measures of heart rate, body sway (i.e., postural freezing), and skin conductance (sympathetic control) were recorded throughout the task. The task was programmed in MATLAB $^{60}$ using the Psychtoolbox ${ }^{61}$ extension. For a detailed description of the trial procedure, see Supplementary Information.

Peripheral stimulation and measurement. Electric shocks were delivered using a 9 V MAXTENS 2000 shocker machine and standard $10 \mathrm{~mm} \mathrm{Ag} / \mathrm{AgCl}$ electrodes which were attached to the distal phalanges of the fourth and fifth fingers of the left hand. Electrocardiogram (ECG), bodily displacement, and electrodermal activity were recorded using a BrainAMP EXG MR 16 channel amplifier, an EXG aux device, and BrainVision Recorder software. To measure heart rate, we made an ECG using two standard $3 \mathrm{M}$ Red Dot $2249 \mathrm{Ag} / \mathrm{AgCl}$ measurement electrodes (diameter of $6.1 \mathrm{~cm}$ each) placed just below the right collar bone and around the lowest left rib (i.e., diagonally through the heart), and a third ground electrode positioned just below the left collar bone (i.e., above the heart). We measured body sway by having participants stand on an in-house built $50 \times 50 \mathrm{~cm}$ stabilometric force platform that detects the participant's displacement relative to the center of pressure, or body 


\begin{tabular}{|l|l|l|}
\hline Dependent variable & Categorical predictors & Continuous predictors \\
\hline Choice (approach/avoid) & Action context (passive/active) & Money; shocks; bradycardia \\
\hline Response type (passive/active) & Choice (approach/avoid) & Money; shocks; bradycardia \\
\hline Response time & Choice (approach/avoid) & Money; shocks; bradycardia \\
\hline Bradycardia & - & Money; shocks; body sway \\
\hline
\end{tabular}

Table 2. Model specifications of mixed models to test task effects on choice behavior and psychophysiology. Note. Money and shock amounts ranged $1-5$.

sway, in the anterior-posterior and medio-lateral direction. For our analyses, we only considered body sway in the anterior-posterior direction, because medio-lateral sway has been shown to be less sensitive to changes in movement when standing bi-pedal ${ }^{8,62}$. Electrodermal activity was assessed using two standard $10 \mathrm{~mm} \mathrm{Ag/}$ $\mathrm{AgCl}$ electrodes attached to the second and third distal phalanges of the left hand with some non-adhesive gel to improve signal quality. Skin conductance responses were only used to account for sympathetic activity in control analyses. Finally, because of the slow development of the heart rate signal over time, only trials with a long stimulus screen duration (i.e., $\geq 6 \mathrm{~s}, 100$ trials per participant) were included in data analysis. See Supplementary Information for a more detailed description.

Data analysis. Quantification of freeze measures. For our analyses we computed trial-by-trial quantifications of our psychophysiological indices of freezing (i.e., bradycardia and verified by body sway) by extracting per trial the mean (HR) or median (BS) signal over a time window of 5000-6000 ms after trial onset (i.e., right before the earliest possible target movement onset). See Supplementary Information for details.

Behavioral analysis. For our behavioral analyses, we complemented our planned computational modeling approaches with mixed-effects modeling to validate our novel approach-avoidance conflict task. Mixed-effects models are well suited to robustly estimate and statistically test average (fixed) effects, while still controlling for individual differences by estimating random effects. Computational modeling (see section below) was used to perform model comparison of different models that included freezing as a parameter. Additionally, parameter estimates of the winning model were correlated to threat-induced bradycardia. All mixed and computational modeling results reported in the main text were robust after controlling for sympathetic activity (i.e., skin conductance), depression and anxiety scores (i.e., BDI- $\mathrm{II}^{59}$ and $\mathrm{STAI}^{58}$ ), and gender (binary; see Supplementary Information for detailed analysis methods).

We ran three separate (generalized) Bayesian mixed-effects models to test task effects on choice [approach/ avoid], response type [passive/active], and response times (see Table 2 for all predictors per model). Because Bayesian analyses do not yield traditional $p$-values, effects were considered 'significant' if the $95 \%$ credible interval (CI) of the posterior distributions did not include 0 . We additionally report $p p$ (i.e., posterior probability) values which are defined as the proportion of the posterior parameter distributions that lies above or below 0 (depending on the direction of the effect) ${ }^{63}$. To be able to interpret them as two-tailed tests that directly reflect the significance of the $95 \%$ CI, $p p$-values are significant when they are smaller than $0.025^{64}$. For all parameter estimates we used the default priors. All Bayesian mixed-effects models followed a maximal random effects structure unless otherwise specified (i.e., by-participant random intercepts, by-participant random slopes for all within-subjects effects, and random correlations for all combinations of random intercepts and slopes ${ }^{65}$; see Supplementary Information for details on priors and model estimation settings).

Computational model. The effect of trial-by-trial potential outcomes (i.e., money and shock amounts) on approach-avoidance choices was formalized according to the following decision value function:

$$
\text { Decision value }(D V)=m^{\alpha_{m}}-s^{\alpha_{s}}+\beta_{m: s}\left(m^{\alpha_{m}} \cdot s^{\alpha_{s}}\right) \text {, }
$$

with exponential free parameters $\alpha_{\mathrm{m}}$ and $\alpha_{\mathrm{s}}$ modulating the effect of the varying money $(\mathrm{m})$ and shock (s) amounts, and free parameter $\beta_{\mathrm{m}: \mathrm{s}}$ weighting their interaction. Since the decision value function in Eq. (1) estimates only the influence of the subjective value (SV) of the potential outcomes on choices without taking into account freezing and the action context, it is further referred to as the SV model ${ }^{34}$. To test whether freezing interacted with the subjective value of the choice or with the action context, this model was then extended via stepwise inclusion of additional parameters of interest that capture the contribution of psychophysiological freezing responses (bradycardia weighted by multiplicative parameter $\beta_{\mathrm{HR}}$ ), individuals' passive-active action tendencies (action context parameter AC), and their interactions (see Table 1). For simplicity, in interaction terms the subjective value of outcomes was operationalized as simply the difference between the money and shock levels $\left(m^{a_{m}}-s^{a_{s}}\right)$. The interaction between, for example, the subjective value and action context parameter AC is thus formalized as $\left(m^{\alpha_{m}}-s^{\alpha_{s}}\right) \cdot A C$.

The action parameter $\mathrm{AC}$ is defined as

$$
\mathrm{AC}=\left\{\begin{array}{ll}
\tau, & \text { if action context }=\text { passive approach } \\
-\tau, & \text { if action context }=\text { active approach }
\end{array},\right.
$$


and it captures the variance in choice behavior related to active vs. passive responding. A positive AC parameter indicates a stronger tendency for passive responses, whereas a negative AC indicates a tendency to respond actively.

To address our hypotheses, our freezing parameter $\left(\beta_{\mathrm{HR}}\right)$ was included as a main effect and in interaction with the previously mentioned SV and AC terms. Though heart rate (bradycardia) was our main index of freezing and body sway was only intended as a validation method, we also fitted the same models with body sway as an alternative freeze index (weighted by its own parameter $\beta_{\mathrm{BS}}$ ) to check robustness of the results (see Supplementary Results). Together, these two are referred to as 'freezing' but are in fact separate parameters estimated in separate models.

The complete decision value function of each model was transformed into a choice probability by passing it through a soft-max logistic choice rule:

$$
p(\text { approach })=\frac{1}{1+e^{-\theta \cdot D V}},
$$

in which $\theta$ is a sensitivity parameter that modulates the slope of the decision value function in terms of choice stochasticity. While all models were initially fitted with $\theta$ as a free parameter, we re-fitted the models with a representative fixed $\theta$-value of 5 (determined by taking the best fitting model's average $\theta$ across participants) in order to increase model stability and allow more reliable interpretation of the parameter estimates ${ }^{66}$. The model comparison results were unaffected by this change.

Based on our hypotheses that freezing interacts with subjective value computations and/or passive-active action tendencies, we selected candidate models for model comparison that iteratively include parameters that denote main effects of the subjective value and action context, the subjective value-by-action interaction (SV:AC), and freezing (bradycardia or body sway) as a main or interaction effect (Table 1). Specifically, we first created models that separately included freezing in interaction with the subjective value, action, or the SV:AC interaction (models 3-5 of Table 1). Then, we included freezing as a main effect in addition to the subjective value, action, or the SV:AC interaction (models 6-8). Finally, we included a single model in which freezing interacts with all of the subjective value, action, and SV:AC parameters simultaneously (model 9). The SV model as described in Eq. (1) was our main reference model since it closely resembles relevant pre-existing computational models of approach-avoidance conflict decisions ${ }^{34}$ and still retains parsimoniousness (model 2). All model candidates were fitted and subsequently compared using maximum likelihood estimation and the Akaike Information Criterion ${ }^{67}$ (see Supplementary Information for details).

Additionally, parameter estimates of the winning model were correlated to a threat-dependent freeze measure (i.e., threat-induced bradycardia) using Spearman rank correlations (alpha of 0.05) to account for any outlier and non-normality related issues. This was computed as the average difference in heart rate deceleration between low ( $\leq 2$ shocks) and high threat ( $\geq 4$ shocks) conditions, which approximates previous quantifications of freezing that contrast low threat and high threat conditions $s^{6,27}$. For the correlation of this threat-related bradycardia with the subjective value of the potential outcomes, we computed the average subjective value per participant using median money and shock levels of 3 (different values did not affect the correlation). Finally, simulations were performed to verify plausibility of the predictions of the winning model (see Supplementary Information). All computational modeling, correlation, and simulation analyses were performed in R 3.6.1 ${ }^{68}$ and RStudio ${ }^{69}$ using various packages $^{70-78}$.

\section{Data availability}

The data sets that support the findings of the current study and the code used to generate them are available in the Donders Institute repository: https://doi.org/10.34973/er3c-0e60.

Received: 16 December 2020; Accepted: 17 May 2021

Published online: 08 June 2021

\section{References}

1. Blanchard, D. C., Griebel, G., Pobbe, R. \& Blanchard, R. J. Risk assessment as an evolved threat detection and analysis process. Neurosci. Biobehav. Rev. 35, 991-998 (2011).

2. Kozlowska, K., Walker, P., McLean, L. \& Carrive, P. Fear and the defense cascade: clinical implications and management. Harv. Rev. Psychiatry 23, 263-287 (2015).

3. Critchley, H. D., Nagai, Y., Gray, M. A. \& Mathias, C. J. Dissecting axes of autonomic control in humans: insights from neuroimaging. Auton. Neurosci. Basic Clin. 161, 34-42 (2011).

4. Critchley, H. D. \& Garfinkel, S. Neural correlates of fear: insights from neuroimaging. Neurosci. Neuroecon. https://doi.org/10. 2147/nan.s35915 (2014).

5. Azevedo, R. T., Garfinkel, S. N., Critchley, H. D. \& Tsakiris, M. Cardiac afferent activity modulates the expression of racial stereotypes. Nat. Commun. 8, 1-9 (2017).

6. Hashemi, M. M. et al. Neural dynamics of shooting decisions and the switch from freeze to fight. Sci. Rep. 9, 1-10 (2019).

7. Niermann, H. C. M., Figner, B. \& Roelofs, K. Individual differences in defensive stress-responses: the potential relevance for psychopathology. Curr. Opin. Behav. Sci. 14, 94-101 (2017).

8. Roelofs, K., Hagenaars, M. A. \& Stins, J. Facing freeze: Social threat induces bodily freeze in humans. Psychol. Sci. 21, 1575-1581 (2010).

9. Lang, P. J., Davis, M. \& Öhman, A. Fear and anxiety: animal models and human cognitive psychophysiology. J. Affect. Disord. 61, 137-159(2000).

10. Carrive, P. The periaqueductal gray and defensive behavior: functional representation and neuronal organization. Behav. Brain Res. 58, 27-47 (1993). 
11. Roelofs, K. Freeze for action: neurobiological mechanisms in animal and human freezing. Philos. Trans. R. Soc. B Biol. Sci. 372, 20160206 (2017).

12. Van Der Zee, E. A., Roozendaal, B., Bohus, B., Koolhaas, J. M. \& Luiten, P. G. M. Muscarinic acetylcholine receptor immunoreactivity in the amygdala-I. Cellular distribution correlated with fear-induced behavior. Neuroscience 76, 63-73 (1997).

13. Nijsen, M. J. M. A. et al. Endogenous corticotropin-releasing hormone inhibits conditioned-fear-induced vagal activation in the rat. Eur. J. Pharmacol. 389, 89-98 (2000).

14. Rosen, J. B. The neurobiology of conditioned and unconditioned fear: a neurobehavioral system analysis of the amygdala. Behav. Cognit. Neurosci. Rev. 3, 23-41 (2004).

15. Bolles, R. C. Species-specific defense reactions and avoidance learning. Psychol. Rev. 77, 32-48 (1970).

16. Bradley, M. M., Codispoti, M., Cuthbert, B. N. \& Lang, P. J. Emotion and motivation I: defensive and appetitive reactions in picture processing. Emotion 1, 276-298 (2001).

17. Blanchard, D. C. Translating dynamic defense patterns from rodents to people. Neurosci. Biobehav. Rev. 76, $22-28$ (2017).

18. Hagenaars, M. A., Oitzl, M. \& Roelofs, K. Updating freeze: aligning animal and human research. Neurosci. Biobehav. Rev. 47, $165-176$ (2014).

19. Bradley, M. M. Natural selective attention: orienting and emotion. Psychophysiology 46, 1-11 (2009).

20. Mobbs, D., Hagan, C. C., Dalgleish, T., Silston, B. \& Prévost, C. The ecology of human fear: survival optimization and the nervous system. Front. Neurosci. 9, 55 (2015).

21. Silva, C. \& McNaughton, N. Are periaqueductal gray and dorsal raphe the foundation of appetitive and aversive control? A comprehensive review. Prog. Neurobiol. 177, 33-72 (2019).

22. Tovote, P. et al. Midbrain circuits for defensive behaviour. Nature 534, 206-212 (2016).

23. Calhoon, G. G. \& Tye, K. M. Resolving the neural circuits of anxiety. Nat. Neurosci. 18, 1394-1404 (2015).

24. Schipper, P. et al. The association between serotonin transporter availability and the neural correlates of fear bradycardia. Proc. Natl. Acad. Sci. U.S.A. 116, 25941-25947 (2019).

25. Lojowska, M., Gladwin, T. E., Hermans, E. J. \& Roelofs, K. Freezing promotes perception of coarse visual features. J. Exp. Psychol. Gen. 144, 1080-1088 (2015).

26. Lojowska, M., Ling, S., Roelofs, K. \& Hermans, E. J. Visuocortical changes during a freezing-like state in humans. Neuroimage 179, 313-325 (2018).

27. Gladwin, T. E., Hashemi, M. M., van Ast, V. \& Roelofs, K. Ready and waiting: freezing as active action preparation under threat. Neurosci. Lett. 619, 182-188 (2016).

28. Charpentier, C. J., Aylward, J., Roiser, J. P. \& Robinson, O. J. Enhanced risk aversion, but not loss aversion, unmedicated pathological anxiety. Biol. Psychiatry 81, 1014-1022 (2017).

29. Tversky, A. \& Kahneman, D. Advances in prospect theory: cumulative representation of uncertainty. J. Risk Uncertain. 5, 297-323 (1992).

30. FeldmanHall, O., Glimcher, P., Baker, A. L. \& Phelps, E. A. Emotion and decision-making under uncertainty: physiological arousal predicts increased gambling during ambiguity but not risk. J. Exp. Psychol. Gen. 145, 1255-1262 (2016).

31. Bach, D. R. Anxiety-like behavioural inhibition is normative under environmental threat-reward correlations. PLoS Comput. Biol. 11, 1-20 (2015).

32. Delgado, M. R., Jou, R. L. \& Phelps, E. A. Neural systems underlying aversive conditioning in humans with primary and secondary reinforcers. Front. Neurosci. 5, 1-10 (2011).

33. Bublatzky, F., Alpers, G. W. \& Pittig, A. From avoidance to approach: the influence of threat-of-shock on reward-based decision making. Behav. Res. Ther. 96, 47-56 (2017).

34. Park, S. Q., Kahnt, T., Rieskamp, J. \& Heekeren, H. R. Neurobiology of value integration: when value impacts valuation. J. Neurosci. 31, 9307-9314 (2011).

35. Kirlic, N., Young, J. \& Aupperle, R. L. Animal to human translational paradigms relevant for approach avoidance conflict decision making. Behav. Res. Ther. 96, 14-29 (2017).

36. Berns, G. S., Capra, C. M., Chappelow, J., Moore, S. \& Noussair, C. Nonlinear neurobiological probability weighting functions for aversive outcomes. Neuroimage 39, 2047-2057 (2008).

37. Ly, V., Huys, Q. J. M., Stins, J. F., Roelofs, K. \& Cools, R. Individual differences in bodily freezing predict emotional biases in decision making. Front. Behav. Neurosci. 8, 237 (2014).

38. Wendt, J., Löw, A., Weymar, M., Lotze, M. \& Hamm, A. O. Active avoidance and attentive freezing in the face of approaching threat. Neuroimage 158, 196-204 (2017).

39. Krypotos, A. M., Effting, M., Kindt, M. \& Beckers, T. Avoidance learning: A review of theoretical models and recent developments. Front. Behav. Neurosci. 9, 189 (2015).

40. Moscarello, J. M. \& LeDoux, J. E. Active avoidance learning requires prefrontal suppression of amygdala-mediated defensive reactions. J. Neurosci. 33, 3815-3823 (2013).

41. Martinez, R. C. R. et al. Active vs. reactive threat responding is associated with differential c-Fos expression in specific regions of amygdala and prefrontal cortex. Learn. Mem. 20, 446-452 (2013).

42. Pavlova, I. V., Rysakova, M. P., Zaichenko, M. I. \& Broshevitskaya, N. D. Behavior of rats with high and low levels of freezing in defensive situations and on selection of food reinforcement. Neurosci. Behav. Physiol. 50, 126-136 (2020).

43. Hashemi, M. M. et al. Human defensive freezing is associated with acute threat coping, long term hair cortisol levels and trait anxiety. bioRxiv https://doi.org/10.1101/554840 (2019).

44. Azevedo, T. M. et al. A freezing-like posture to pictures of mutilation. Psychophysiology 42, 255-260 (2005).

45. Niermann, H. C. M., Figner, B., Tyborowska, A., Cillessen, A. H. N. \& Roelofs, K. Investigation of the stability of human freezinglike responses to social threat from mid to late adolescence. Front. Behav. Neurosci. 12, 97 (2018).

46. Lehrer, P. et al. Heart rate variability biofeedback improves emotional and physical health and performance: a systematic review and meta analysis. Appl. Psychophysiol. Biofeedback 45, 109-129 (2020).

47. Brammer, J. C. et al. Breathing biofeedback for police officers in a stressful virtual environment: challenges and opportunities. Front. Behav. Neurosci. 12, 401 (2021).

48. Bramson, B., den Ouden, H., Toni, I. \& Roelofs, K. Improving emotional-action control by targeting long-range phase-amplitude neuronal coupling. Elife 9, 1-19 (2020).

49. Fendt, M. et al. Context and trade-offs characterize real-world threat detection systems: a review and comprehensive framework to improve research practice and resolve the translational crisis. Neurosci. Biobehav. Rev. 115, 25-33 (2020).

50. Bramson, B. et al. Human lateral frontal pole contributes to control over emotional approach-avoidance actions. J. Neurosci. 40, 2925-2934 (2020).

51. Eder, A. B. \& Hommel, B. Anticipatory control of approach and avoidance: An ideomotor approach. Emot. Rev. 5, 275-279 (2013).

52. Frijda, N. H., Ridderinkhof, K. R. \& Rietveld, E. Impulsive action: emotional impulses and their control. Front. Psychol. 5, 1-10 (2014).

53. Niermann, H. C. M. et al. The relation between infant freezing and the development of internalizing symptoms in adolescence: a prospective longitudinal study. Dev. Sci. 22, 1-10 (2019).

54. Guitart-Masip, M., Duzel, E., Dolan, R. \& Dayan, P. Action versus valence in decision making. Trends Cognit. Sci. 18, 194-202 (2014). 
55. Green, A. L. et al. Stimulating the human midbrain to reveal the link between pain and blood pressure. Pain 124, 349-359 (2006).

56. Green, P. \& McLeod, C. J. SIMR: an R package for power analysis of generalised linear mixed models by simulation. Methods Ecol. Evol. 7, 493-498 (2016).

57. Chen, H., Cohen, P. \& Chen, S. How big is a big odds ratio? Interpreting the magnitudes of odds ratios in epidemiological studies. Commun. Stat. Simul. Comput. 39, 860-864 (2010).

58. Spielberger, C. D., Gorsuch, R. L., Lushene, R., Vagg, P. R. \& Jacobs, G. A. Manual for the State-Trait Anxiety Inventory (Consulting Psychologists Press, 1983).

59. Beck, A. T., Steer, R. A. \& Brown, G. K. Manual for the Beck Depression Inventory-II (Psychological Corporation, 1996).

60. The Mathworks. MATLAB (2018).

61. Brainard, D. H. The psychophysics toolbox. Spat. Vis. 10, 433-436 (1997).

62. Niermann, H. C. M. et al. Infant attachment predicts bodily freezing in adolescence: evidence from a prospective longitudinal study. Front. Behav. Neurosci. 9, 1-10 (2015).

63. Maier, S. U., Raja Beharelle, A., Polanía, R., Ruff, C. C. \& Hare, T. A. Dissociable mechanisms govern when and how strongly reward attributes affect decisions. Nat. Hum. Behav. 4, 949-963 (2020).

64. Makowski, D., Ben-Shachar, M. S., Chen, S. H. A. \& Lüdecke, D. Bayesian indices of existence and significance. Front. Psychol. 10, 1-14 (2019).

65. Barr, D., Levy, R., Scheepers, C. \& Tily, H. J. Random effects structure for confirmatory hypothesis testing: keep it maximal. J. Mem. Lang. 68, 1-43 (2013).

66. Gershman, S. J. Empirical priors for reinforcement learning models. J. Math. Psychol. 71, 1-6 (2016).

67. Akaike, H. A new look at the statistical model identification. IEEE Trans. Autom. Contr. 19, 716-723 (1974).

68. R Core Team. R: A Language and Environment for Statistical Computing (2019).

69. RStudio Team. RStudio: Integrated Development for R (2019).

70. Bürkner, P.-C. Advanced Bayesian multilevel modeling with the R package brms. RJ. 10, 395-411 (2018).

71. Bürkner, P.-C. brms: An R package for Bayesian multilevel models using stan. J. Stat. Softw. 80, 1-28 (2017).

72. Bolker, B. \& R Core Team. bbmle: Tools for General Maximum Likelihood Estimation (2017).

73. Wickham, H. ggplot2: Elegant Graphics for Data Analysis (2016).

74. Fox, J. \& Weisberg, S. An $\{R\}$ Companion to Applied Regression, 3rd edn. (2019).

75. Attali, D. \& Baker, C. ggExtra: Add Marginal Histograms to 'ggplot2', and More 'ggplot2' Enhancements (2019).

76. Pedersen, T. L. patchwork: The Composer of Plots (2019).

77. Neuwirth, E. RColorBrewer: ColorBrewer Palettes (2014).

78. Stan Development Team. RStan: the R interface to Stan (2019).

\section{Acknowledgements}

This work was supported by a consolidator grant from the European Research Council (ERC_CoG 2017_772337) awarded to KR.

\section{Author contributions}

F.H.K., L.H., B.F., J.X.O., and K.R. conceived and designed the study, F.H.K. and L.H. collected the data, F.H.K. performed the data analysis, all authors were involved in data interpretation, F.H.K. wrote the first full draft, F.H.K., L.D.d.V., and K.R. edited the manuscript, and all authors contributed to the final manuscript.

\section{Competing interests}

The authors declare no competing interests.

\section{Additional information}

Supplementary Information The online version contains supplementary material available at https://doi.org/ 10.1038/s41598-021-90968-z.

Correspondence and requests for materials should be addressed to F.H.K.

Reprints and permissions information is available at www.nature.com/reprints.

Publisher's note Springer Nature remains neutral with regard to jurisdictional claims in published maps and institutional affiliations.

(c) (i) Open Access This article is licensed under a Creative Commons Attribution 4.0 International License, which permits use, sharing, adaptation, distribution and reproduction in any medium or format, as long as you give appropriate credit to the original author(s) and the source, provide a link to the Creative Commons licence, and indicate if changes were made. The images or other third party material in this article are included in the article's Creative Commons licence, unless indicated otherwise in a credit line to the material. If material is not included in the article's Creative Commons licence and your intended use is not permitted by statutory regulation or exceeds the permitted use, you will need to obtain permission directly from the copyright holder. To view a copy of this licence, visit http://creativecommons.org/licenses/by/4.0/.

(C) The Author(s) 2021 